\title{
MICROSECOND-SCALE ELECTRIC FIELD PULSES IN CLOUD LIGHTNING FLASHES
}

\author{
Y. Villanueva, V.A. Rakov, M.A. Uman \\ Electrical Engineering Department, University of Florida, Gainesville, Florida \\ M. Brook \\ New Mexico Institute of Mining and Technology, Socorro, New Mexico
}

From wideband electric field records acquired using a 12-bit digitizing system with a 500-ns sampling interval, microsecond-scale pulses in different stages of cloud flashes in Florida and New Mexico are analyzed. The pulse occurrence statistics and waveshape characteristics are presented. The larger pulses tend to occur carly in the flash and presumably are related to the flashinitiating breakdown processes. This result contradicts the common view found in the atmospheric VLF radio noise literature (e.g., [1]) that the main sources of electromagnetic radiation in cloud flashes are $\mathrm{K}$ processes which occur in the final, or J-type, part of the cloud flash.

\section{Introduction}

Recently, Bils et al. [2], from an analysis of taperecorded wideband electric fields of close cloud flashes in Florida, reported that relatively large pulses with a width (at half peak value) of a few microseconds occur predominantly in the initial part of the flash, as opposed to its final, or J-type, part during which step-like, millisecond-scale $\mathrm{K}$ electric field changes (see also $[3,4]$ ) are often observed. Less than 20 percent of $\mathrm{K}$ field changes were found to contain microsecond-scale pulses at least 50 percent above the system noise level. The Kpulses were relatively small and irregular in waveshape. A similar lack of pronounced pulse activity associaled with $\mathrm{K}$ changes was reported (also from tape-recorded data) for ground flashes by Rakov et al. [5]. Note that the $K$ process in a lightning flash, i.e., the physical process producing the $\mathrm{K}$ change, has long been considered in theoretical atmospheric VLF radio-noise studies as the main source of electromagnetic radiation in cloud flashes and in ground flashes as a source second only to return strokes (e.g., [1]). All of the initial cloudflash pulses observed by Bils et al. [2] were of negative polarity, opposite to the polarity of a return stroke lowering negative charge to ground (atmospheric electricity sign convention [6]).

One potential problem in interpreting the results of Bils et al. [2] is associated with the inherent analog tape noise which could have affected their measured pulse occurrence statistics. To verify the generality of the previous results, we here examine low-noise electric field records acquired with a 12-bit digitizing system (see, for example, [7]) allowing a 500-ns sampling interval with individual record lengths up to a few seconds. The analysis of these better-quality data allows us to study the cloud-flash pulse occurrence statistics as a function of not only the time elapsed from the beginning of the flash but also of the pulse magnitude. Further, for the first time, we compare the microsecond-scale pulse activity associated with cloud flashes in three geographical locations as recorded with the same measurement system: in Socorro, New Mexico, at the NASA Kennedy Space Center (KSC), Florida, and in Gainesville, Florida. The results of this study, besides providing additional insights into the physics of the lightning discharge, can have important implications for lightning-related EMC problems.

\section{Data}

Electric field data (see Table 1) were acquired with a system composed of a flat-plate antenna, an integrator (the decay time constant being $10 \mathrm{~s}$ or $150 \mu \mathrm{s}$ ), a low pass anti-aliasing filter (rise time of about $0.7 \mu \mathrm{s}$ ), and a 12-bit $2 \mathrm{MHz}$ digitizer. Following the terminology introduced by Kitagawa and Brook [3], we call the system configuration with the decay time constant of 10 $s$ the Slow Antenna and the system configuration with the decay time constant of $150 \mu \mathrm{s}$ the Fast Antenna. The Slow Antenna had a useful bandwidth of about $0.1 \mathrm{~Hz}$ to $1 \mathrm{MHz}$.

All records analyzed here are longer than $500 \mathrm{~ms}$ and contain both early and final, or J-type, portions [3]. No reliable information on the distance to the recorded discharges is available. In Figs. 1, 2, and 3 (top portions) we show examples of the overall electric field changes (as recorded by the Slow Antenna) due to cloud discharges from Socorro, KSC, and Gainesville, respectively. The field changes are initially negativegoing (opposite in polarity to the field change due to a cloud-to-ground discharge lowering negative charge to ground). The pulse occurrence statistics presented here have been derived from the Slow Antenna records. It is worth noting that many of the Slow Antenna records (particularly from KSC and Gainesville), presumably at relatively close ranges, contain no pronounced pulse activity. Most likely, the pulses do exist but are undetectable due to being overwhelmed by the electrostatic field changes, dominant at closer ranges. Those pulseless records are not considered here. The 
Table 1. Summary of the Data Analyzed

\begin{tabular}{|c|c|c|c||}
\hline Geographical Location & Socorro, New Mexico & KSC, Florida & Gainesville, Florida \\
\hline Time Period & August, 1989 & July and August, 1991 & September, 1991 \\
\hline Measurement System & Slow Antenna & Slow and Fast Antennas & Slow Antenna \\
\hline Number of Flashes & 8 & $4^{*}$ & 2 \\
\hline
\end{tabular}

* Three flashes were recorded simultaneously by the Slow and Fast Antennas; one flash was recorded by the Slow Antenna only.

pulse waveshape characteristics were measured in the Fast Antenna records which provide better reproduction of the microsecond scale features than the Slow Antenna records. In Figs. $4 \mathrm{a}$ and $4 \mathrm{~b}$ we show examples of typical single-peaked and multiple-peaked pulses, respectively, as recorded by the Fast Antenna.

\section{Results and Discussion}

We separated the cloud-flash pulses found in the Slow Antenna records into four categories depending on relative pulse magnitude within the flash. The pulse categories, labeled Large, Medium, Small, and Tiny, were arbitrarily determined in the following way. We first found the average peak-to-peak amplitude $\mathrm{A}$ of the five largest pulses in the flash. A Large pulse is any pulse with a peak-to-peak amplitude greater than or equal to $0.5^{*} \mathrm{~A}$; a Medium pulse is any pulse with a peak-topeak amplitude greater than or equal to $0.25^{*} \mathrm{~A}$, but less than $0.5^{*} \mathrm{~A}$; and a Small pulse is any pulse with a peakto-peak amplitude greater than or equal to $0.125 * \mathrm{~A}$, but less than $0.25 * \mathrm{~A}$. Other pulses that were too small to fit in the Small pulse category, but had amplitudes noticeably higher than the noise level were labeled Tiny pulses. The latter are not considered here. In the Gainesville data (two flashes only) it was impossible to sort pulses by their size using the algorithm described above due to the very narrow range of the observed pulse magnitudes.

In Figs. 1, 2, and 3 we show, for each of the three pulse-magnitude categories (when possible), the histogram of the pulse occurrence distribution on the same time scale as the corresponding overall cloud-flash field change given in the top portion of each Figure. The field changes and histograms are representative of the entire data set. A summary of the occurrence statistics for the I arge pulses is given in Table 2 where the "early stage" is arbitrarily defined as the first $80 \mathrm{~ms}$ after the initial deflection of the electric field from the preceding more-or-less flat level, the rest of the discharge being termed the "late stage". In most cases the first maximum of the Slow-Antenna field change is attained within the so-defined early stage (see Figs. 1 and 2). The "entire flash" in Table 2 is merely the sum of the early and late stages.

\section{Pulse occurrence statistics}

The larger pulses usually appear at the times of the largest changes in the cloud-flash electric field. In most cases this corresponds to the first deflection of the field from the preceding flat level. The observation that larger pulses tend to occur early in the flash suggests the relation of those pulses to the flash-initiating breakdown processes. It is important to note that about 60 percent of the Large pulses are found within the first $20 \mathrm{~ms}$ of the flash (lasting typically for some hundreds of milliseconds) and are of the same polarity as the initial cloud flash field change. This result is consistent with the observations of Bils et al. [2] and with the cloud-channel formation time of 10 to $30 \mathrm{~ms}$ reported by Liu and Krehbiel [8]. Pierce [9] also reported that radiation pulses associated with cloud flashes at ranges of 40 to $100 \mathrm{~km}$ in Great Britain were usually confined to the first 10 to $20 \mathrm{~ms}$ of the flash. Our results further suggest an even narrower time interval for the largest pulse occurrence: about 50 percent of the Large pulses appeared within the first $5 \mathrm{~ms}$ of the flash.

In the Gainesville data (see Fig. 3), the majority of pulses occurred, consistent with the occurrence of Large pulses in the Socorro and KSC data, during the first few tens of milliseconds of the flash. However, the two Gainesville flashes analyzed exhibited, in contrast with the Socorro and KSC data, some pulse activity preceding the main pulse burst. These preliminary pulses are probably related to the initial, relatively slow ramp preceding the most prominent variation in the flash field change. Such a slow initial ramp was not observed in the Socorro and KSC cloud flashes. Since it was not possible to categorize the Gainesville pulses by size, the Gainesville data are not included in Table 2.

Less than 30 percent of the Large pulses occurred during the late stage of the flash, where the relatively small magnitude $\mathbf{K}$ field changes are usually observed to occur [2-4]. In many cases the subsidiary burst of Large pulses corresponded to a sudden, relatively-large increase in the slope of the flash field change, perhaps due to the onset of a new in-cloud breakdown process. The fact that the larger pulses tend to avoid the late stage of the flash (although the occurrence of smaller pulses may be largest there) indicates that $\mathrm{K}$-change pulses are smaller, if detectable at all, than pulses occurring in the early part of the cloud discharge.

The results of the present study strongly support the previously reported [2-4] difference between the early and late parts of the cloud discharge. However, we did not find any distinction which would allow us to separate the early part of our cloud flash records into the initial and very active stages introduced by Kitagawa and Brook [3]. In fact, the main burst of Large pulses almost invariably marked the beginning of the cloud flash. Note that Kitagawa and Brook [3] stressed the distinction only between the early and late stages and exercised caution that "the difference between the initial and very active portions is not always well marked, the transition from 
Table 2. Occurrence of Large Pulses in Different Parts of Cloud Flash

\begin{tabular}{|c|c|c|c|c|c|c|}
\hline \multicolumn{7}{|c|}{ Socorro, New Mexico, 1989} \\
\hline \multirow{3}{*}{ Day } & \multirow{3}{*}{ Flash } & \multicolumn{5}{|c|}{ Number of Pulses Within } \\
\hline & & \multicolumn{3}{|c|}{ Early Stage } & \multirow{2}{*}{$\begin{array}{l}\text { Late } \\
\text { Stage }\end{array}$} & \multirow{2}{*}{$\begin{array}{l}\text { Entire } \\
\text { Flash }\end{array}$} \\
\hline & & First $5 \mathrm{~ms}$ & First $20 \mathrm{~ms}$ & First $80 \mathrm{~ms}$ & & \\
\hline 242 & 76 & 9 & 9 & 10 & 0 & 10 \\
\hline 246 & 50 & 6 & 6 & 10 & 4 & 14 \\
\hline 250 & 6 & 2 & 3 & 4 & 13 & 17 \\
\hline 250 & 7 & 4 & 4 & 6 & 3 & 9 \\
\hline 250 & 8 & 7 & 8 & 8 & 1 & 9 \\
\hline 250 & 9 & 7 & 9 & 10 & 0 & 10 \\
\hline 250 & $1 \dot{2}$ & 5 & 6 & 7 & 2 & 9 \\
\hline 250 & 13 & 6 & 6 & 8 & 0 & 8 \\
\hline \multicolumn{2}{|c|}{ Total Pulses } & 46 & 51 & 63 & 23 & 86 \\
\hline \multicolumn{2}{|c|}{$\begin{array}{l}\text { Average Number of } \\
\text { Pulses (Percentage) }\end{array}$} & $5.8(53)$ & $6.4(59)$ & $7.9(73)$ & $2.9(27)$ & $11(100)$ \\
\hline \multicolumn{7}{|c|}{ KSC, Florida, 1991} \\
\hline \multirow{3}{*}{ Day } & \multirow{3}{*}{ Flash } & \multicolumn{5}{|c|}{ Number of Pulses Within } \\
\hline & & \multicolumn{3}{|c|}{ Early Stage } & \multirow{2}{*}{$\begin{array}{l}\text { Late } \\
\text { Stage }\end{array}$} & \multirow{2}{*}{$\begin{array}{l}\text { Entire } \\
\text { Flash }\end{array}$} \\
\hline & & First $5 \mathrm{~ms}$ & First $20 \mathrm{~ms}$ & First $80 \mathrm{~ms}$ & & \\
\hline 205 & 37 & 6 & 6 & 6 & 0 & 6 \\
\hline 225 & 297 & 2 & 6 & 7 & 7 & 14 \\
\hline 231 & 64 & 6 & 9 & 9 & 0 & 9 \\
\hline 236 & 6 & 3 & 4 & 9 & 0 & 9 \\
\hline \multicolumn{2}{|c|}{ 'Total Pulses } & 17 & 25 & 31 & 7 & 38 \\
\hline \multicolumn{2}{|c|}{$\begin{array}{l}\text { Average Number of } \\
\text { Pulses (Percentage) }\end{array}$} & $4.3(45)$ & $6.3(66)$ & $7.8(82)$ & $1.8(18)$ & $9.5(100)$ \\
\hline
\end{tabular}

Table 3. Characteristics of Large Pulses

\begin{tabular}{|c|c|c|c|}
\hline Waveshape Parameter & Sample Size & Mean Value & Standard Deviation \\
\hline Total Pulse Width Including Overshoot, $\mu \mathrm{s}$ & 22 & 56 & 35 \\
\hline Full Width of the Initial Peak, $\mu \mathrm{s}$ & 22 & 26 & 11 \\
\hline Initial Peak Width at Half Value, $\mu \mathrm{s}$ & 22 & 3.3 & 2.3 \\
\hline Ratio of Initial Peak to Overshoot & 19 & 6.4 & 4.3 \\
\hline
\end{tabular}




\section{Socorro 1989 Day 250 Flash 12}
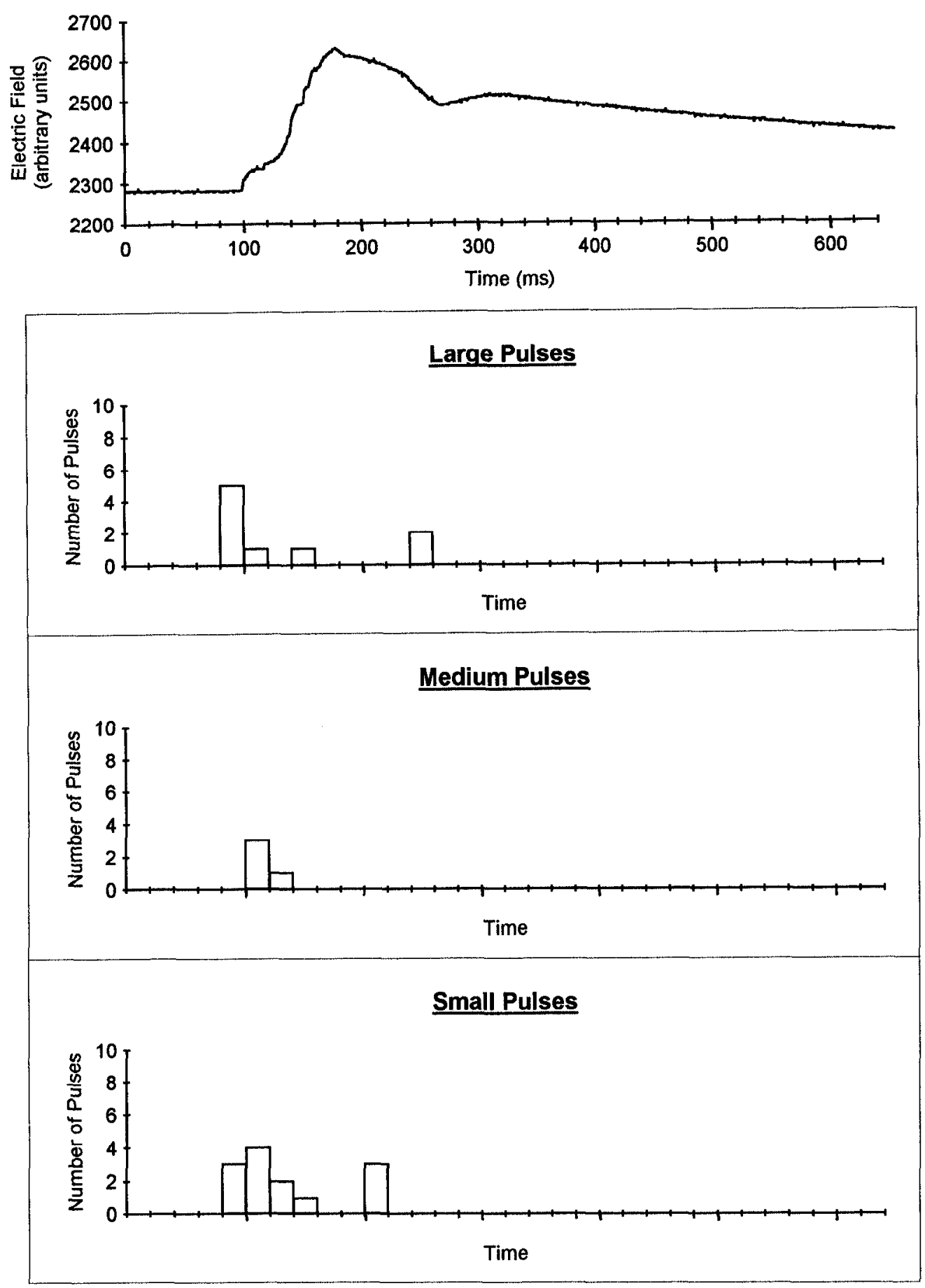

Fig. 1. Histograms of the occurrence of Large, Medium, and Small pulses (defined in the text) in different parts of a cloud flash in Socorro, New Mexico, whose electric field change is shown in the top portion of the Figure. The field change and histograms are displayed on the same time scale. Negative field change (atmospheric electricity sign convention) deflects upward. 
one to the other usually being more or less gradual" and that in more than 40 percent of cases the initial portion was absent. Ogawa and Brook [4] considered the same process to be responsible for both the initial and very active stages and called the entirety of those two "the initial part of the discharge", as opposed to its final part.

\section{Pulse waveshape characteristics}

A summary of the waveshape characteristics of representative Large pulses from three cloud flashes that occurred in three different thunderstorms at KSC is presented in Table 3. All the pulses were negative, 16 of them having single-peaked and 6 multiple-peaked waveshapes (see examples given in Fig. 4). We did not attempt to determine the $30-90 \%$ [2] or $10-90 \%$ [10] rise times of the pulses, because these times were expected to be comparable to the digitizer sampling interval of 0.5 $\mu \mathrm{s}$.

KSC 1991 Day 231 Flash 64


Fig. 2. Similar to Fig. 1, but for a flash at KSC, Florida. 


\section{Gainesville 1991 Day 267 Flash 41}
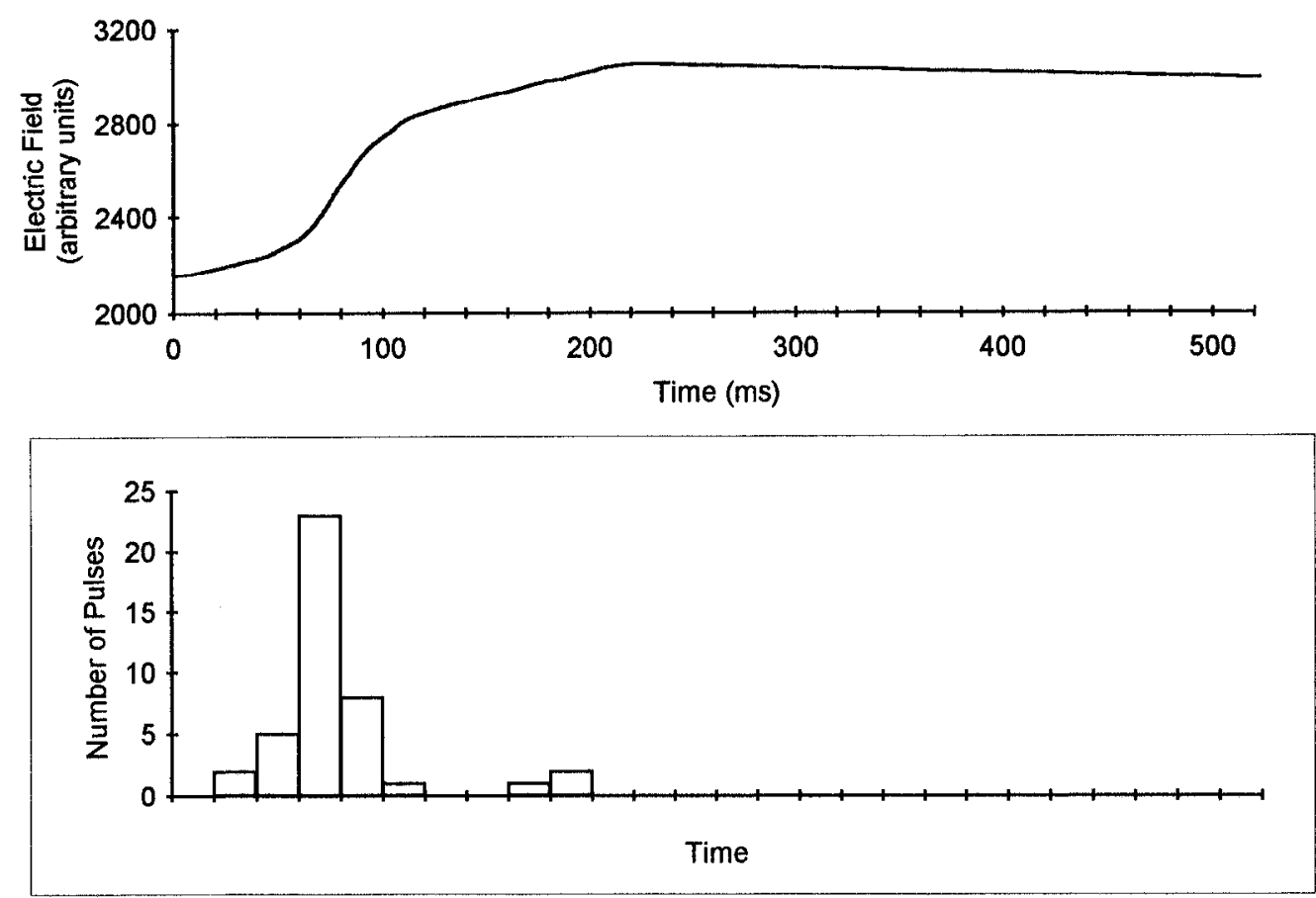

Fig. 3. Similar to Fig. 1, but for a flash in Gainesville, Florida, with all pulses being combined in a single category.

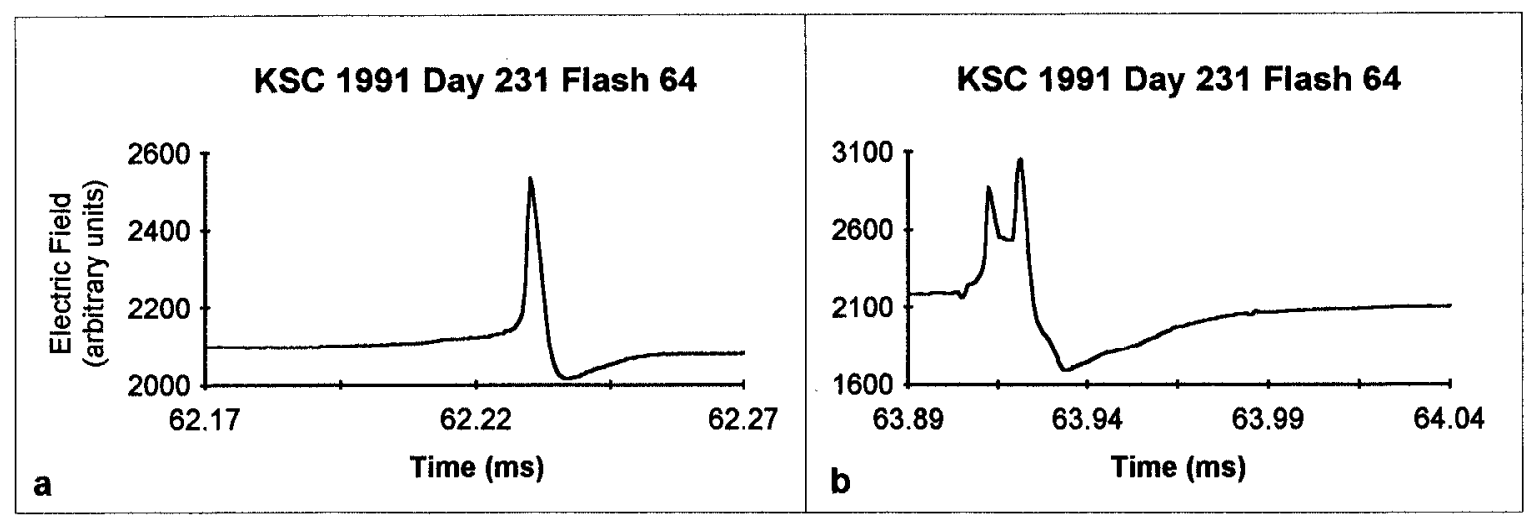

Fig. 4. Examples of typical single-peaked (a) and multiple-peaked (b) cloud-flash pulses. Both pulses belong to the Large pulse category and occurred within the first $5 \mathrm{~ms}$ of the cloud discharge whose overall field change is shown in Fig. 2. Negative field change (atmospheric electricity sign convention) deflects upward.

Acknowledgement. This research has been funded in part by the National Science Foundation grant ATM-9014085 and by the Russian State Committee for Science and Higher Education, grant Tom-VN-18. The authors wish to thank K. Risa Altaf for her assistance with the data analysis.

\section{$\underline{\text { References }}$}

1. Arnold, H.R. and E.T. Pierce: Radio Sci., 68D, 771776 (1964).

2. Bils, J.R., E.M. Thomson, M.A. Uman, and D. Mackerras: J. Geophys. Res., 93, 15,933-15,940 (1988).

3. Kitagawa, N. and M. Brook: J. Geophys. Res., 65, 1189-1201 (1960).
4. Ogawa, T. and M. Brook: J. Geophys. Res., $\underline{69}$, 5141-5150 (1964).

5. Rakov, V.A., R. Thottappillil, and M.A. Uman: J. Geophys. Res., 97, 9935-9950 (1992).

6. Uman, M.A.: The lightning discharge, Academic Press, San Diego (1987).

7. Brook, M.: Res. Lett. Atmos. Electr., 12, 47-52 (1992).

8. Liu, X.-S. and P.R. Krehbiel: J. Geophys. Res., 90, 6211-6218 (1985).

9. Pierce, E.T.: Quart. J. Roy. Met. Soc., $\underline{81}$, 211-228 (1955).

10. Medelius, P.J., E.M. Thomson, and J.S Pierce: Proc. 1991 Int. Aerospace and Ground Conf. on Lightning and Static Electricity, Vol. I, (1991). 